\title{
Five transiting hot Jupiters discovered using WASP-South, Euler, and TRAPPIST: WASP-119 b, WASP-124 b, WASP-126 b, WASP-129 b, and WASP-133 $b^{\star}$
}

P. F. L. Maxted ${ }^{1}$, D. R. Anderson ${ }^{1}$, A. Collier Cameron ${ }^{2}$, L. Delrez ${ }^{3}$, M. Gillon ${ }^{3}$, C. Hellier ${ }^{1}$, E. Jehin ${ }^{3}$, M. Lendl ${ }^{4,5}$, M. Neveu-VanMalle ${ }^{5,6}$, F. Pepe ${ }^{5}$, D. Pollacco ${ }^{7}$, D. Queloz ${ }^{6,5}$, D. Ségransan ${ }^{5}$, B. Smalley ${ }^{1}$, A. M. S. Smith ${ }^{1,8}$, J. Southworth ${ }^{1}$, A. H. M. J. Triaud ${ }^{5,9,10}$, S. Udry ${ }^{5}$, T. Wagg ${ }^{1}$, and R. G. West ${ }^{7}$

1 Astrophysics Group, Keele University, Staffordshire, ST5 5BG, UK e-mail: p.maxted@keele.ac.uk

2 SUPA, School of Physics and Astronomy, University of St. Andrews, North Haugh, Fife, KY16 9SS, UK

3 Institut d'Astrophysique et de Géophysique, Université de Liège, Allée du 6 Août, 17, Bât. B5C, Liège 1, Belgium

${ }^{4}$ Space Research Institute, Austrian Academy of Sciences, Schmiedlstr. 6, 8042 Graz, Austria

5 Observatoire Astronomique de l'Université de Genève, 51 ch. des Maillettes, 1290 Sauverny, Switzerland

${ }^{6}$ Cavendish Laboratory, J J Thomson Avenue, Cambridge, CB3 OHE, UK

7 Department of Physics, University of Warwick, Coventry CV4 7AL, UK

8 Institute of Planetary Research, German Aerospace Center, Rutherfordstrasse 2, 12489 Berlin, Germany

9 Centre for Planetary Sciences, University of Toronto at Scarborough, 1265 Military Trail, Toronto, ON, M1C 1A4, Canada

${ }^{10}$ Department of Astronomy \& Astrophysics, University of Toronto, Toronto, ON, M5S 3H4, Canada

Received 4 February 2016 / Accepted 24 April 2016

\section{ABSTRACT}

\begin{abstract}
We have used photometry from the WASP-South instrument to identify 5 stars showing planet-like transits in their light curves. The planetary nature of the companions to these stars has been confirmed using photometry from the EulerCam instrument on the Swiss Euler 1.2-m telescope and the TRAPPIST telescope, and spectroscopy obtained with the CORALIE spectrograph. The planets discovered are hot Jupiter systems with orbital periods in the range 2.17 to 5.75 days, masses from $0.3 M_{\text {Jup }}$ to $1.2 M_{\text {Jup }}$ and with radii from $1 R_{\mathrm{Jup}}$ to $1.5 R_{\mathrm{Jup}}$. These planets orbit bright stars $(V=11-13)$ with spectral types in the range F9 to G4. WASP-126 is the brightest planetary system in this sample and hosts a low-mass planet with a large radius $\left(0.3 M_{\text {Jup }}, 0.95 R_{\text {Jup }}\right)$, making it a good target for transmission spectroscopy. The high density of WASP-129 A suggests that it is a helium-rich star similar to HAT-P-11 A. WASP$133 \mathrm{~A}$ has an enhanced surface lithium abundance compared to other old G-type stars, particularly other planet host stars. These planetary systems are good targets for follow-up observations with ground-based and space-based facilities to study their atmospheric and dynamical properties.
\end{abstract}

Key words. planetary systems

\section{Introduction}

Ground-based transit surveys such as WASP (Pollacco et al. 2008), HAT (Bakos et al. 2004), HAT-South (Bakos et al. 2013) and KELT (Pepper et al. 2008) have identified the majority of bright hot Jupiter planetary systems that have been studied in detail using observations with large ground-based telescopes and space-based instrumentation. Although space-based surveys such as Kepler-K2 (Howell et al. 2014), TESS (Ricker et al. 2015) and PLATO (Rauer et al. 2014) will produce much higher quality photometry than is possible from the ground, it is advantageous to identify as many planetary systems as possible in the areas of the sky that will be observed by these missions so that the observing strategy can be optimised. This was clearly demonstrated by the recent discovery that WASP47 is a very rare example of a multiple-planet system containing a hot Jupiter (Becker et al. 2015; Neveu-VanMalle et al. 2016). This discovery was only possible because this known

\footnotetext{
* Full Tables 2 and 3 are only available at the CDS via anonymous ftp to cdsarc.u-strasbg. fr (130.79.128.5) or via http://cdsarc.u-strasbg.fr/viz-bin/qcat?]/A+A/591/A55
}

planetary system was prioritised for high-cadence observations with Kepler-K2. These high-cadence observations made it possible to detect the transit time variations produced by the gravitational interactions between the planets in this system. The discovery that WASP-47 is a multi-planet system also demonstrates that detailed obervations of apparently normal hot Jupiter systems continue to reveal unexpected properties of this diverse group of planetary systems.

Here we present the discovery of 5 hot-Jupiter planetary systems in the southern hemisphere. Section 2 describes the observations we have obtained, Sect. 3 describes our analysis of the host stars, the stellar and planetary masses and radii are derived in Sect. 4 and we discuss the properties of each system briefly in Sect. 5.

\section{Observations}

WASP-South uses an array of 8 cameras that observe selected regions of the southern sky with a combined area of approximately 450 square degrees at a typical cadence of about $8 \mathrm{~min}$ 
Table 1. Log of observations.

\begin{tabular}{|c|c|c|}
\hline Facility & Date & Notes \\
\hline \multicolumn{3}{|l|}{ WASP-119 } \\
\hline WASP-South & 2010 Aug.-2012 Feb. & 14010 points \\
\hline CORALIE & 2013 Sep.-2014 Nov. & 18 spectra \\
\hline CORALIE & 2015 Jan.-2015 Sep. & 5 spectra \\
\hline TRAPPIST & 2013 Nov. 15 & NIR \\
\hline \multicolumn{3}{|l|}{ WASP-124 } \\
\hline WASP-South & 2006 May-2011 Nov. & 15165 points \\
\hline CORALIE & 2011 Dec.-2014 Oct. & 28 spectra \\
\hline CORALIE & 2015 May-2015 Nov. & 13 spectra \\
\hline TRAPPIST & 2012 Nov. 17 & ExoPlanet-BB \\
\hline TRAPPIST & 2013 May 28 & ExoPlanet-BB \\
\hline TRAPPIST & 2013 Jun. 07 & ExoPlanet-BB \\
\hline TRAPPIST & 2013 Aug. 17 & ExoPlanet-BB \\
\hline TRAPPIST & 2013 Nov. 06 & ExoPlanet-BB \\
\hline Euler & 2015 Jul. 16 & NGTS filter \\
\hline \multicolumn{3}{|l|}{ WASP-126 } \\
\hline WASP-South & 2010 Aug.-2012 Feb. & 13245 points \\
\hline CORALIE & 2013 Oct.-2014 Oct. & 22 spectra \\
\hline CORALIE & 2014 Nov.-2015 Sep. & 8 spectra \\
\hline TRAPPIST & 2014 Jan. 08 & $z^{\prime}$ \\
\hline TRAPPIST & 2014 Jan. 31 & $z^{\prime}$ \\
\hline Euler & 2014 Sep. 05 & Gunn $r$ filter \\
\hline Euler & 2014 Oct. 17 & Gunn $r$ filter \\
\hline Euler & 2014 Nov. 06 & Gunn $r$ filter \\
\hline \multicolumn{3}{|l|}{ WASP-129 } \\
\hline WASP-South & 2006 Мау-2012 Мay & 26940 points \\
\hline CORALIE & 2014 Feb.-2014 Jun. & 9 spectra \\
\hline Euler & 2015 Mar. 08 & NGTS filter \\
\hline Euler & 2015 Mar. 31 & $\mathrm{I}_{\mathrm{C}}$ \\
\hline TRAPPIST & 2015 Mar. 31 & NIR \\
\hline \multicolumn{3}{|l|}{ WASP-133 } \\
\hline WASP-South & 2006 May-2012 Jun. & 18436 points \\
\hline CORALIE & 2014 Aug.-2014 Oct. & 12 spectra \\
\hline TRAPPIST & 2014 Sep. 26 & ExoPlanet-BB \\
\hline Euler & 2015 Jun. 14 & NGTS filter \\
\hline TRAPPIST & 2015 Aug. 25 & ExoPlanet-BB \\
\hline
\end{tabular}

Notes. Data obtained after an upgrade to the CORALIE spectrograph in November 2014 are treated separately to data obtained before the upgrade. In the final column we list either the number of observations obtained with WASP-South, or the number of spectra obtained, or the filter used to obtained the transit photometry. The different filters used are described in the text.

and the exposure time is $30 \mathrm{~s}$. The WASP survey and instruments are described in Pollacco et al. (2006) and a description of our planet-hunting methods can be found in Pollacco et al. (2008) and Collier Cameron et al. (2007).

WASP-South planet candidates are followed up using the TRAPPIST robotic photometer (Jehin et al. 2011; Gillon et al. 2011), and the CORALIE spectrograph and EulerCam photometer (Lendl et al. 2012) on the Swiss Euler 1.2-m telescope at La Silla. We find that about 1-in-12 candidates turns out to be a planet, the remainder being blends that are unresolved in the
Table 2. Light curves of five transiting exoplanet systems.

\begin{tabular}{llllrlr}
\hline \hline WASP- & Source & Filter & Date & \multicolumn{1}{c}{$\Delta m$} & \multicolumn{1}{c}{$\sigma_{m}$} & Flip \\
\hline 119 & W & WASP & 5780.52513 & 0.0123 & 0.0163 & 0 \\
119 & W & WASP & 5780.52557 & -0.0135 & 0.0159 & 0 \\
119 & W & WASP & 5780.54147 & -0.0014 & 0.0156 & 0 \\
\hline
\end{tabular}

Notes. This table is available in its entirety at CDS. The first few lines are shown here for guidance as to its contents and format. Column 1 is the WASP identification number of the star. Column 2 indicates the source of the observation as follows: W - WASP-South; T - TRAPPIST; E - EulerCam. Column 3 indicates the filter used for the observations as follows: WASP - 400-700 nm; NIR - Astrodon NIR $700 \mathrm{~nm}$ cut-on luminance; Exo - Astrodon ExoPlanet-BB; NGTS - EulerCam NGTS; $z \_\mathrm{p}-z^{\prime}$; Gunn - Gunn $R$; $I_{-} \mathrm{C}-$ Cousins $I_{\mathrm{C}}$. Column 4 is the UTC date of the observation given as HJD-2 450000 . Columns 5 and 6 are the apparent magnitude of the star and its standard error relative to the mean out-of-transit apparent magnitude. Column 7 is a flag that is set to the value 1 for the first observation after a meridian flip with the TRAPPIST telescope.

Table 3. CORALIE radial velocities.

\begin{tabular}{rrrrr}
\hline \hline \multicolumn{2}{l}{ WASP-119 } & & & \\
\hline WASP- & $\begin{array}{r}\text { BJD(UTC) } \\
-2400000\end{array}$ & $\begin{array}{r}\text { RV } \\
\left(\mathrm{km} \mathrm{s}^{-1}\right)\end{array}$ & $\begin{array}{l}\sigma_{\mathrm{RV}} \\
\left(\mathrm{km} \mathrm{s}^{-1}\right)\end{array}$ & $\begin{array}{r}\text { BS } \\
\left(\mathrm{km} \mathrm{s}^{-1}\right)\end{array}$ \\
\hline 119 & 56548.906910 & 8.45864 & 0.01532 & -0.09451 \\
119 & 56570.857181 & 8.24473 & 0.01756 & 0.01842 \\
119 & 56575.777466 & 8.24146 & 0.01258 & 0.01012 \\
119 & 56593.863562 & 8.42141 & 0.02158 & -0.00951 \\
119 & 56622.794968 & 8.33225 & 0.02814 & -0.05036 \\
\hline
\end{tabular}

Notes. This table is available in its entirety at CDS. The first few lines are shown here for guidance as to its contents and format. Data are provided to the full precision used for calculations, but times of midexposure are only accurate to a few minutes. The standard error on the bisector span (BS) measurement is twice the standard error on the $\mathrm{RV}\left(\sigma_{\mathrm{RV}}\right)$.

WASP images (which have 14 arcsec pixels) or astrophysical transit mimics, usually eclipsing binary stars. A list of observations reported in this paper is given in Table 1 . The light curve data used in our analysis are given in Table 2 and the radial velocities measured from the CORALIE spectra are listed in Table 3. The radial velocity measurements presented in Table 3 use a mask based on a G2-type template for the calculation of the cross-correlation function. We also measured the radial velocity using a K5-type template and found that the resuls are consistent to within $7 \mathrm{~m} \mathrm{~s}^{-1}$. This test excludes most scenarios in which the periodic RV variations we observe are due to an eclipsing binary star blended with the light of the target star (Huélamo et al. 2008). Also given in Table 3 are the bisector span (BS) measurements that characterise the asymmetry in the stellar line profiles for each spectrum (Queloz et al. 2001). The bisector span will show a significant correlation with the measured radial velocity (RV) if the apparent variations in RV are due to stellar magnetic activity (star spots) or blended spectra in a multiple star system. We used the Bayesian information criterion (BIC) to test the significance of any correlation between RV and BS for each star, i.e., we calculated the change of BIC going from a constant $\mathrm{BS}$ value (weighted mean) to a fit of a straight line to $\mathrm{BS}$ as a function of RV. The zero-points of the RV and BS measurements may be slightly different for spectra obtained before or after the 
upgrade to CORALIE in November 2014 so we analysed data either side of this date independently. Weak evidence for a correlation is present in the data obtained prior to the CORALIE upgrade for WASP-124 (BIC decreased by 6.6) but this apparent correlation is not confirmed by the data obtained post-upgrade and there is no other indication that WASP-124 is an active star or a multiple star system, so we assume that this correlation is a statistical fluke. None of the data sets for any other stars show any evidence for a correlation between BS and RV.

To measure precise planetary radii, we obtained follow-up photometry of the transits of these stars with EulerCam and TRAPPIST. The dates of observation and filters used are given in Table 1. The NGTS filter on the EulerCam instrument has a central wavelength of $698 \mathrm{~nm}$ and the effective bandwidth is $312 \mathrm{~nm}$. The ExoPlanet-BB filter manufactured by Astrodon blocks wavelengths below about $500 \mathrm{~nm}$. The NIR filter is an NIR luminance filter also from Astrodon that blocks light below $700 \mathrm{~nm}$.

\section{The host stars}

\subsection{Spectroscopic analysis}

The CORALIE spectra of the host stars were co-added to produce spectra for analysis using the methods described in Gillon et al. (2009) and Doyle et al. (2013). We used the H $\alpha$ line to estimate the effective temperature $\left(T_{\text {eff }}\right)$, and the $\mathrm{Na}$ I D and $\mathrm{Mg}$ I b lines as diagnostics of the surface gravity $(\log g)$. The resulting parameters are listed in Table 4. The CORALIE spectra do not have a sufficient number of clean spectral lines to determine reliable values for $[\mathrm{Mg} / \mathrm{Fe}]$ or $[\mathrm{Na} / \mathrm{Fe}]$ independently of $\log g$ so the quoted error in $\log g$ includes an additional 0.15 dex error due to the unknown $\mathrm{Mg}$ abundance of these stars (Brewer et al. 2015). The iron abundances were determined from equivalent-width measurements of several clean and unblended Fe I lines and are given relative to the solar value presented in Asplund et al. (2009). The quoted abundance errors include that given by the uncertainties in $T_{\text {eff }}$ and $\log g$, as well as the scatter due to measurement and atomic data uncertainties. The projected rotation velocities $(v \sin I)$ were determined by fitting the profiles of the Fe I lines after convolving with the CORALIE instrumental resolution $(R=55000)$ and a macroturbulent velocity adopted from the calibration of Doyle et al. (2014).

\subsection{Rotational modulation}

We searched the WASP photometry of each star for rotational modulations by using a sine-wave fitting algorithm as described by Maxted et al. (2011). We estimated the significance of periodicities by subtracting the fitted transit light curve and then repeatedly and randomly permuting the nights of observation. For none of our stars was a significant periodicity obtained, with 95\%-confidence upper limits on the semi-amplitude being typically $1 \mathrm{mmag}$.

\section{System parameters}

The CORALIE radial-velocity measurements were combined with the WASP, EulerCam and TRAPPIST photometry in a simultaneous Markov-chain Monte-Carlo (MCMC) analysis to find the system parameters. For details of our methods see Collier Cameron et al. (2007). Limb-darkening was parameterised using the 4-parameter non-linear law and coefficients from Claret (2000). The value of $T_{\text {eff }}$ used to interpolate the limb
Table 4. Stellar parameters.

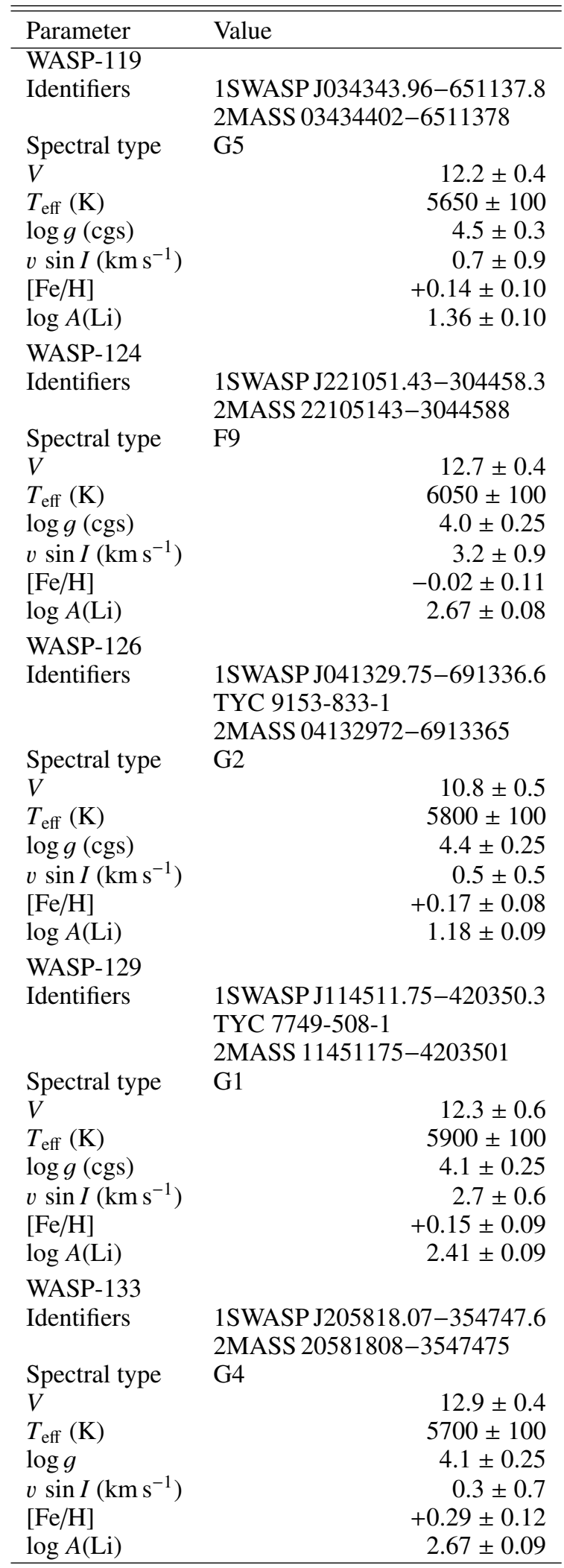

Notes. The $V$ magnitude is based on the estimated apparent Gaia $G$ magnitude from Smart (2013) and the transformation $V-G$ from Jordi et al. (2010) assuming $V-I_{\mathrm{C}} \approx 0.7$ for all stars. Other symbols are defined in the text. The 1 SWASP identifier provides the J2000.0 coordinates of the star taken from the USNO-B1.0 catalogue (Monet et al. 2003). 
Table 5. Stellar mass and age estimates.

\begin{tabular}{lll}
\hline \hline Star & Mass $\left[M_{\odot}\right]$ & Age $[\mathrm{Gyr}]$ \\
\hline WASP-119A & $1.02 \pm 0.06(1.01)$ & $8.0 \pm 2.5(8.1)$ \\
WASP-124 A & $1.07 \pm 0.05(1.10)$ & $2.1 \pm 1.4(1.1)$ \\
WASP-126 A & $1.12 \pm 0.06(1.10)$ & $6.4 \pm 1.6(6.4)$ \\
WASP-129 A $^{a}$ & $1.00 \pm 0.03(1.03)$ & $1.0 \pm 0.9(0.0)$ \\
WASP-133 A & $1.16 \pm 0.08(1.20)$ & $6.8 \pm 1.8(5.7)$ \\
\hline
\end{tabular}

Notes. The mean and standard deviation of the posterior distributions are given together with the best-fit values in parentheses. ${ }^{(a)}$ Values for helium-enhanced models, $\Delta Y=+0.02$.

darkening coefficients from these tables is a free parameter in the fit but is constrained by a Gaussian prior according to value of $T_{\text {eff }}$ and its standard error for each star from Table 4 . The resulting values of $T_{\text {eff }}$ are all found to be consistent with prior values so we do not quote them here. We used the coefficients for the $R$-band to model the WASP light curves and those obtained with the ExoPlanet-BB, NGTS and Gunn $R$ filters. For the $z^{\prime}$ and NIR filters we used the coefficients for the Sloan $z^{\prime}$-band from Claret (2004). The coefficients for $\log g=4.5,[\mathrm{M} / \mathrm{H}]=0.1$ and micro-turbulence velocity $V_{\mathrm{T}}=2 \mathrm{~km} \mathrm{~s}^{-1}$ were interpolated from these tables for each trial value of the effective temperature in the MCMC chain. Changing the choice of table used to calculate the limb darkening coefficients assigned to these non-standard filters from $z^{\prime}$ to $R$-band or from $R$-band to $I$-band changes the mass and radius derived for the stars and planets by less than the quoted uncertainty. For TRAPPIST observations where a meridian flip was required the data obtained with the telescope on the west side and east side of the German equatorial mount were analysed independently to allow for any change in the photometric zero-point. To account for stellar noise in the radial velocity measurements ("jitter") we used a circular orbit fit by least-squares with the orbital period and time of mid-transit fixed to values determined from the photometry. We then used trial-and-error to find the amount of jitter required to achieve a reduced $\chi^{2}$ value of 1 when this additional variance is added in quadrature to the standard errors given in Table 3 . The values of the jitter $\left(\sigma_{\mathrm{jit}}\right)$ used in our analysis are given in Table 6 . We did not find strong evidence in any of these systems for a linear drift in the apparent centre-of-mass velocity, $\frac{\mathrm{d} \gamma}{\mathrm{d} t}$. There is weak evidence for a drift in the velocity of WASP-126 A $\left(\frac{\mathrm{d} \gamma}{\mathrm{d} t}=(5.5 \pm 3.0) \times 10^{-5} \mathrm{~km} \mathrm{~s}^{-1} \mathrm{~d}^{-1}\right)$, but we do not consider this to be a significant detection. Adding this extra parameter in the modelling of the data changes the other parameters by less than one standard deviation.

For all of our planets the data are compatible with zero eccentricity and hence we imposed a circular orbit - the rationale for this assumption is discussed in Anderson et al. (2012). The fitted parameters were thus $T_{\mathrm{c}}, P, \Delta F, T_{14}, b, K_{1}$, where $T_{\mathrm{c}}$ is the epoch of mid-transit, $P$ is the orbital period, $\Delta F$ is the planet-star area ratio (i.e., the fractional flux-deficit that would be observed during transit in the absence of limb-darkening), $T_{14}$ is the total transit duration (from first to fourth contact), $b$ is the impact parameter of the planet's path across the stellar disc, and $K_{1}$ is the stellar reflex velocity semi-amplitude.

The analysis of the transit light curves leads directly to an estimate of the stellar density (Seager \& Mallén-Ornelas 2003) and the spectroscopic orbit can then be used to infer the planetary surface gravity (Southworth et al. 2007). One additional constraint is required to obtain the masses and radii of the star and planet. The additional constraint we have used is the mass

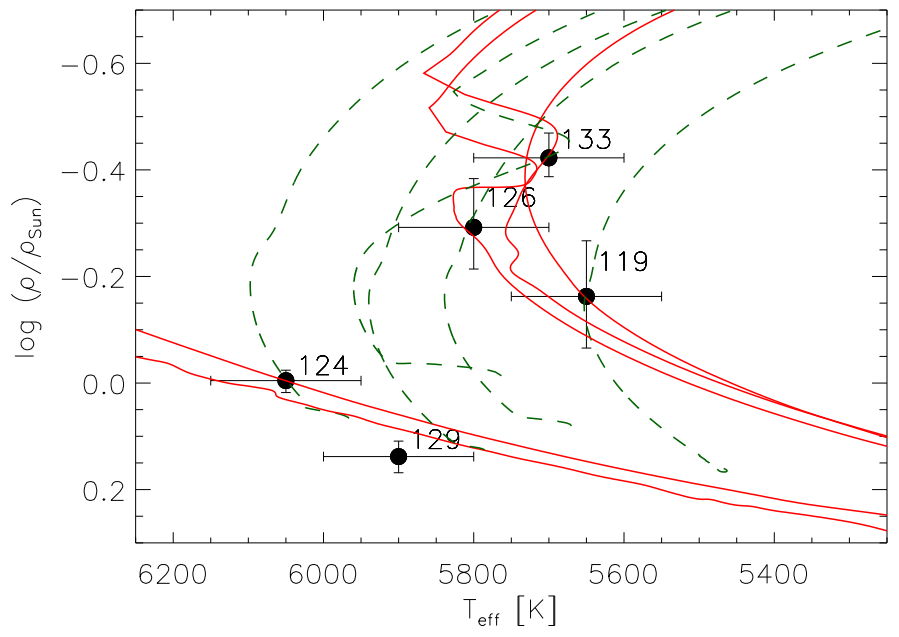

Fig. 1. Planet host stars in the $T_{\text {eff }}$ - density plane (error bars labelled by WASP identification number) compared to best-fit evolution tracks (dashed lines) and isochrones (solid lines) for the masses and ages listed in Table 5 assuming $[\mathrm{Fe} / \mathrm{H}]$ as listed in Table 4.

of the star estimated using stellar models based on the effective temperature, metallicity and mean density of the star. We used the open source software BAGEMASs ${ }^{1}$ to calculate the posterior mass distribution for each star using the Bayesian method described by Maxted et al. (2015a). The models used in BAGEMASS were calculated using the GARSTEC stellar evolution code (Weiss \& Schlattl 2008). The mean and standard deviation of the posterior mass distribution for the star are included in the MCMC analysis as a Gaussian prior. The mass and age of the stars derived are shown in Table 5. There is no good match to the observed density of WASP-129 A if we use the standard grid of stellar models in BAGEMASS with the following linear relation between helium abudance, $Y$ and heavy element abundance, $Z$ : $Y=0.2485+0.984 Z$, where the zero-point in this relation is the primordial helium abundance (Steigman 2010) and the gradient is set to match the current solar helium abundance. For this star we used the grid of stellar models provided within BAGEMASS with an enhanced initial helium abundance $(\Delta Y=+0.02)$. This has the effect of reducing the mass estimate for this star by about one standard deviation. The best-fit stellar evolution tracks and isochrones are shown in Fig. 1.

The parameters for each planetary system derived from our analysis are given in Table 6 . The discovery data and model fits to these observations are shown in Figs. 2-6.

\subsection{Kinematics}

The kinematics of our sample in Galactic coordinates are summarized in Table 7. The proper motions are taken from Smart (2013) and the radial velocities are from Table 6. To calculate the distance we use the apparent $K_{\mathrm{s}}$-band magnitude from Skrutskie et al. (2006), the radius of the star from Table 6 and the angular diameter of the star based on the calibration of $K$-band surface brightness - effective temperature relation from Kervella et al. (2004). We assume that interstellar reddening is negligible and that $K=K_{\mathrm{s}}+0.044$ (Bessell 2005). The transformation of these variables to Galactic $(U, V, W)$ velocity in the local standard of rest is done using the procedure gal_uvw ${ }^{2} . U$ is

\footnotetext{
http://sourceforge.net/projects/bagemass
}

idlastro.gsfc.nasa.gov 
P. F. L. Maxted et al.: Five transiting hot Jupiters
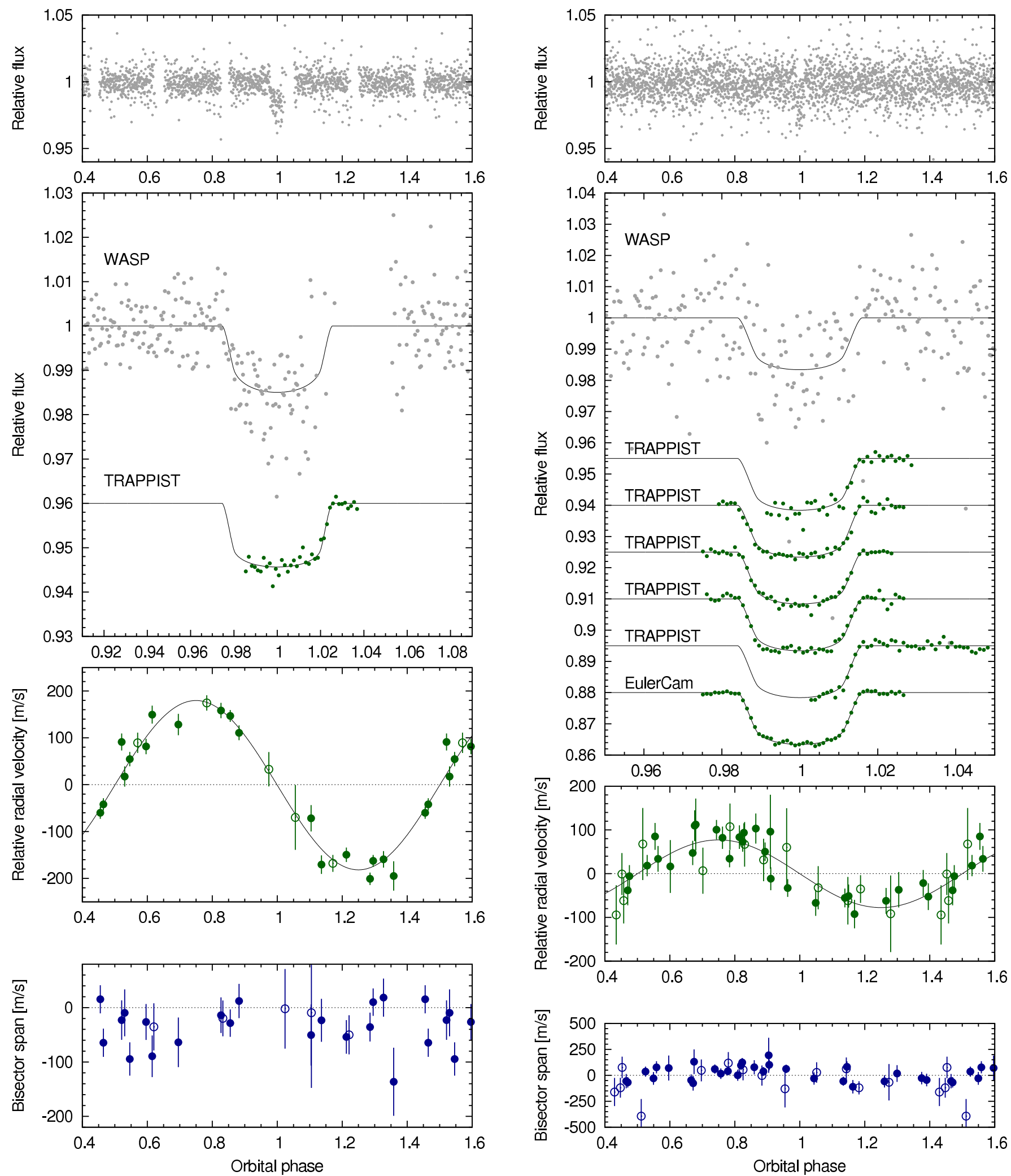

Fig. 2. WASP-119b discovery data: (top) the WASP-South light curve folded on the transit period. (Second panel) The binned WASP data with (offset) the follow-up transit light curves (ordered from top-tobottom as in Table 1) together with the fitted MCMC model. (Third) The CORALIE radial velocities with the fitted model. Filled/open symbols denote data obtained before/after the upgrade to the CORALIE spectrograph, respectively. (Lowest) The bisector spans; the absence of any significant correlation with radial velocity is a check against transit mimics.
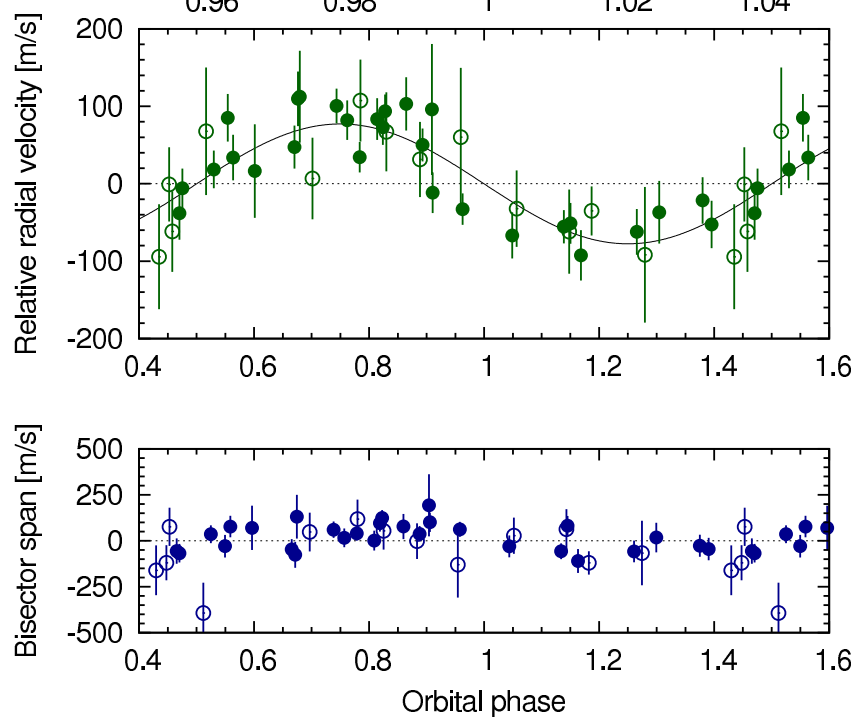

Fig. 3. WASP-124 b discovery data, as for Fig. 1.

positive towards the Galactic anti-centre and the solar motion is taken from Coşkunoğlu et al. (2011).

Holmberg et al. (2007) find that individual stars in the solar neighbourhood cannot be unambiguously assigned to different populations based only on their kinematics, but the welldefined age - velocity dispersion relation for solar-type stars 

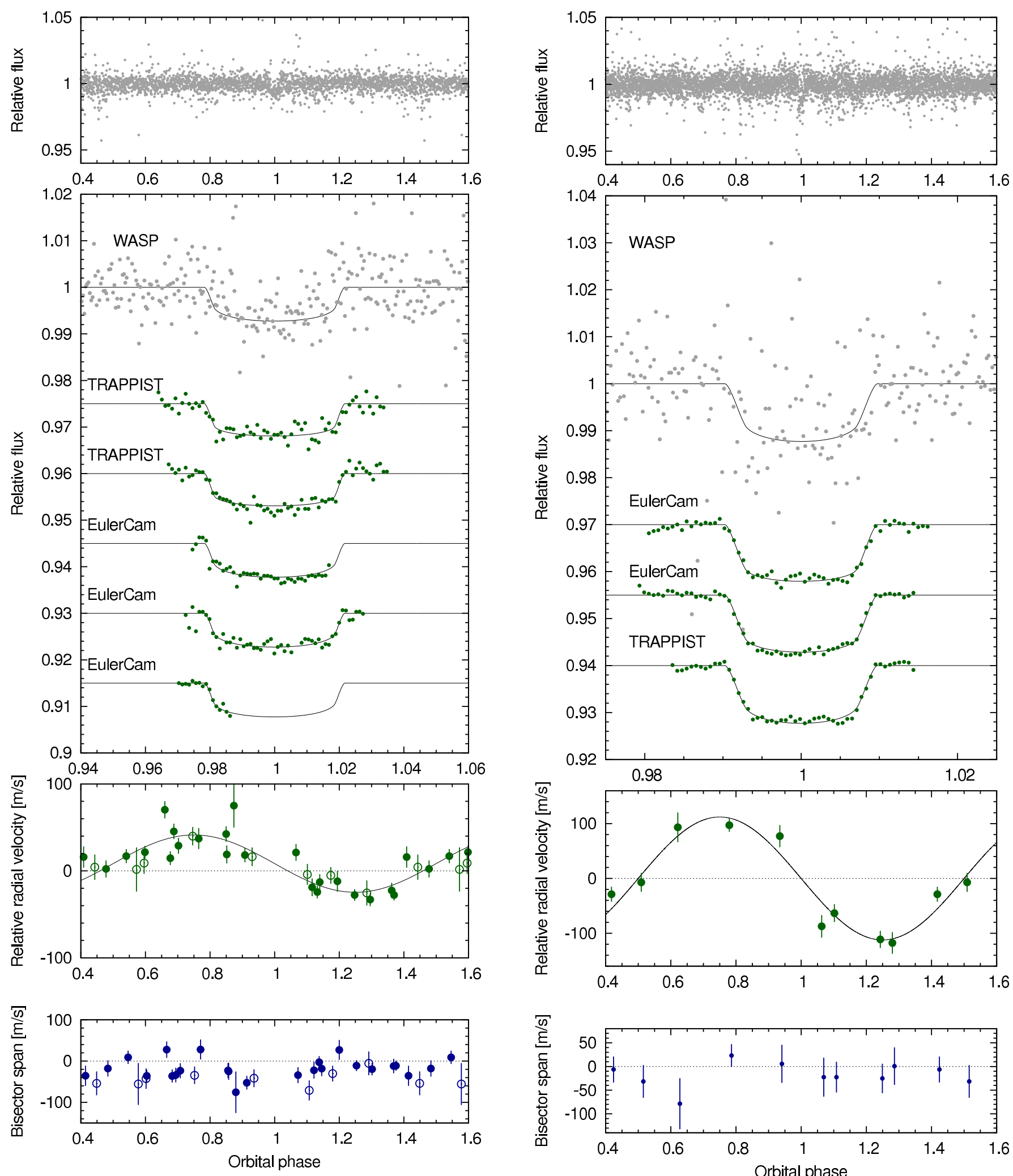

Fig. 4. WASP-126b discovery data, as for Fig. 1.

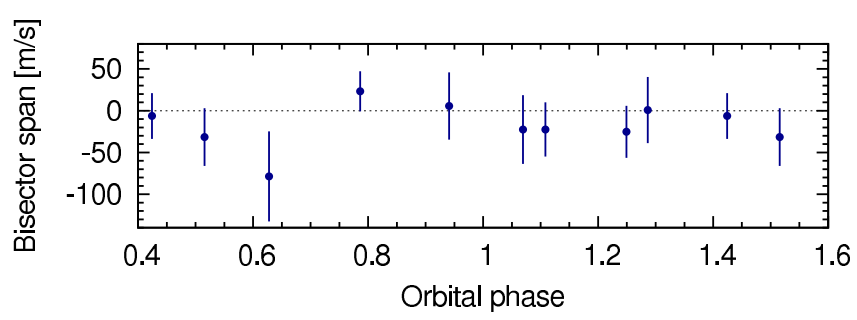

Fig. 5. WASP-129 b discovery data, as for Fig. 1.

\section{Discussion}

does suggest that WASP-126 A is likely to be older than about 4 Gyr based on its kinematics, consistent with the age of about 6 Gyr we have derived using stellar models.

WASP-119 is a typical hot Jupiter system in terms of the mass (1.2 $\left.M_{\text {Jup }}\right)$, radius $\left(1.3 R_{\text {Jup }}\right)$ and orbital period $(2.5 \mathrm{~d})$ of the planet. The host star has a similar mass to the Sun but appears 

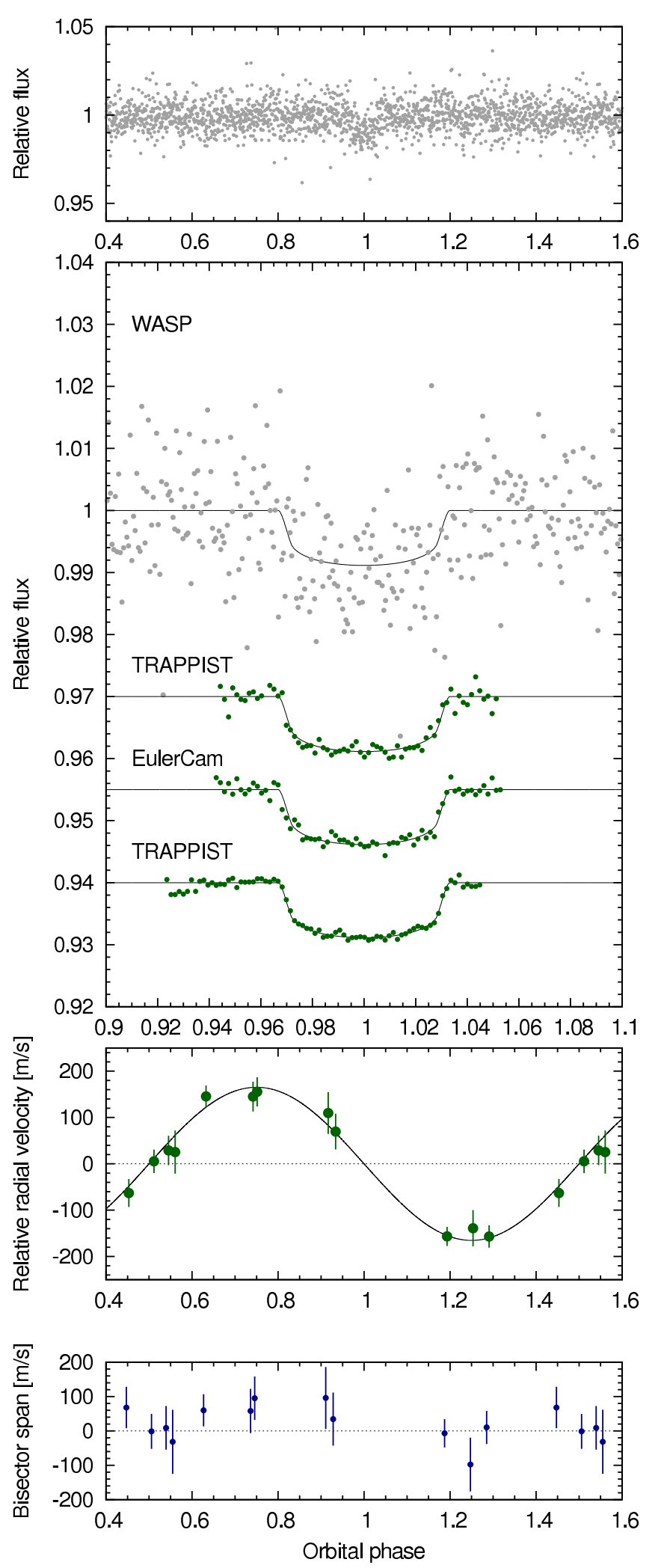

Fig. 6. WASP-133 b discovery data, as for Fig. 1.

to be much older based on its effective temperature and density (Fig. 1).

WASP-124 is also a typical hot Jupiter system (0.6 $M_{\text {Jup }}$, $1.2 R_{\text {Jup }}, 3.4$ d) but with an apparently much younger host star, although not so young that it has an enhanced lithium abundance. The projected rotation velocity of WASP-124 A is one of the largest in our sample ( $v \sin I \approx 3 \mathrm{~km} \mathrm{~s}^{-1}$ ). Rapid rotation is expected for young solar-type stars, but it is unclear whether the age estimates for planet host stars based on their rotation rate (gyrochronological ages) are reliable (Maxted et al. 2015b).

WASP-126 A is the brightest star presented here and also hosts the lowest-mass planet in our sample $\left(0.3 \mathrm{M}_{\text {Jup }}\right)$. The radius of this planet is quite large $\left(0.95 R_{\text {Jup }}\right)$ so it also has the lowest surface gravity of these newly-discovered planets. This combination of low surface gravity (i.e., large atmospheric scale height) and a bright host star make this a good target for transmission spectroscopy.

The high density of WASP-129 A can be explained using stellar models with enhanced initial helium abudance. The helium-enhanced models we have used here $(\Delta \mathrm{Y}=+0.02)$ provide an adequate fit to the observed properties of this star, so we expect that models with an even higher helium abundance $(\Delta \mathrm{Y} \approx+0.04)$ would provide a much better fit. Maxted et al. (2015b) found similarly high densities for two other hot Jupiter host stars (HAT-P-11 A and WASP-84 A). These high densities inferred from the transit light curves cannot be explained by contamination of the light curve by a third star in the system since this would lead to a shallower eclipse depth and a lower inferred value for the stellar density (Seager \& Mallén-Ornelas 2003). The distribution of the initial helium abundance for solar-type stars is very uncertain since there is no direct way to measure the helium abundance of cool stars, so it is unclear how this parameter impacts on our understanding of the age and mass distributions for planet host stars. The surface gravity of WASP-129 A derived from our analysis of the light curves and spectroscopic orbit $(4.53 \pm 0.02)$ is clearly inconsistent with the value derived from our analysis of its spectrum $(4.1 \pm 0.1)$. Analysis of the spectrum of WASP-129 A at high resolution and higher signalto-noise may help to find the reason for this discrepancy. WASP$129 \mathrm{~b}$ has a slightly longer orbital period than most hot Jupiters (5.7 d) and, for the stellar mass that we have adopted, also has a smaller radius $\left(0.9 R_{\text {Jup }}\right)$ than most hot Jupiters with similar masses $\left(1.0 M_{\text {Jup }}\right)$. The high density estimate for WASP-129 b implied by these parameters should be treated with some caution until the mass of the host star can be determined with greater confidence. The surface gravity of this planet is also high compared to other hot Jupiters and this quantity is independent of the assumed stellar mass.

WASP-133 A appears to be an old, metal-rich G-dwarf when compared to stellar evolution models (Fig. 1). WASP-133 b is a hot Jupiter $\left(1.2 M_{\text {Jup }}, 1.2 R_{\text {Jup }}\right)$ with the shortest orbital period of the systems presented here $(2.2 \mathrm{~d})$. The surface lithium abundance of WASP-133 A $(A(\mathrm{Li})=2.7)$ is significantly higher than other stars of similar age and effective temperature $(A(\mathrm{Li})<2.5$, Sestito \& Randich 2005). This is a counter-example of the general trend for planet host stars to be depleted in lithium compared to similar non-planet host stars (Maxted et al. 2010; Gonzalez 2015; Deal et al. 2015). Whether this anomaly is connected to the slow rotation of this star or some other factor is difficult to ascertain from the consideration of a single system. Understanding the relationships between these different properties of planets and their host star can only be done by studying a large sample of planetary systems, such as the one being compiled using WASPSouth of which these new discoveries are the latest contribution.

Acknowledgements. WASP-South is hosted by the South African Astronomical Observatory and we are grateful for their ongoing support and assistance. Funding for WASP comes from consortium universities and from the UK's Science and Technology Facilities Council. TRAPPIST is funded by the Belgian Fund for Scientific Research (Fond National de la Recherche Scientifique, FNRS) under the grant FRFC 2.5.594.09.F, with the participation of the Swiss National 
Table 6. System parameters.

\begin{tabular}{lrrrrr}
\hline \hline Parameter & WASP-119 & WASP-124 & WASP-126 & WASP-129 & WASP-133 \\
\hline$P(\mathrm{~d})$ & $2.49979(1)$ & $3.372650(1)$ & $3.28880(1)$ & $5.748145(4)$ & $2.176423(1)$ \\
$T_{\mathrm{c}}$ & $6537.547(2)$ & $7028.5834(1)$ & $6890.3191(6)$ & $7027.4373(2)$ & $7116.9874(2)$ \\
$T_{14}(\mathrm{~d})$ & $0.126 \pm 0.006$ & $0.1071 \pm 0.0006$ & $0.142 \pm 0.002$ & $0.1119 \pm 0.0008$ & $0.1433 \pm 0.0007$ \\
$\Delta F$ & $0.0131 \pm 0.0008$ & $0.0154 \pm 0.0002$ & $0.0061 \pm 0.0002$ & $0.0114 \pm 0.0001$ & $0.0074 \pm 0.0001$ \\
$b$ & $0.50_{-0.24}^{+0.10}$ & $0.61 \pm 0.02$ & $0.3 \pm 0.2$ & $0.62 \pm 0.02$ & $0.2 \pm 0.1$ \\
$i\left(^{\circ}\right)$ & $85 \pm 2$ & $86.3 \pm 0.2$ & $87.9 \pm 1.5$ & $87.7 \pm 0.2$ & $87 \pm 1$ \\
$R_{\star} / a$ & $0.158 \pm 0.015$ & $0.106 \pm 0.018$ & $0.131_{-0.004}^{+0.011}$ & $0.066 \pm 0.0017$ & $0.194_{-0.003}^{+0.006}$ \\
$K_{1}\left(\mathrm{~km} \mathrm{~s}^{-1}\right)$ & $0.181 \pm 0.010$ & $0.077 \pm 0.008$ & $0.036 \pm 0.005$ & $0.11 \pm 0.01$ & $0.17 \pm 0.01$ \\
$\gamma\left(\mathrm{km} \mathrm{s}^{-1}\right)$ & $8.404 \pm 0.007$ & $-5.876 \pm 0.006$ & $29.164 \pm 0.004$ & $21.988 \pm 0.008$ & $-23.913 \pm 0.009$ \\
$M_{\star}\left(M_{\odot}\right)$ & $1.02 \pm 0.06$ & $1.07 \pm 0.05$ & $1.12 \pm 0.06$ & $1.00 \pm 0.03$ & $1.16 \pm 0.08$ \\
$R_{\star}\left(R_{\odot}\right)$ & $1.2 \pm 0.1$ & $1.02 \pm 0.02$ & $1.27+0.10$ & $0.90 \pm 0.02$ & $1.44 \pm 0.05$ \\
$\log g_{\star}(\mathrm{cgs})$ & $4.26 \pm 0.08$ & $4.44 \pm 0.02$ & $4.28+0.03$ & $4.53 \pm 0.02$ & $4.18 \pm 0.02$ \\
$\rho_{\star}\left(\rho_{\odot}\right)$ & $0.54 \pm 0.18$ & $0.99 \pm 0.05$ & $0.56_{-0.12}^{+0.06}$ & $1.38 \pm 0.10$ & $0.39 \pm 0.03$ \\
$M_{\mathrm{P}}\left(M_{\mathrm{Jup}}\right)$ & $1.23 \pm 0.08$ & $0.60 \pm 0.07$ & $0.28 \pm 0.04$ & $1.0 \pm 0.1$ & $1.16 \pm 0.09$ \\
$R_{\mathrm{P}}\left(R_{\mathrm{Jup}}\right)$ & $1.4 \pm 0.2$ & $1.24 \pm 0.03$ & $0.96+0.10$ & $0.93 \pm 0.03$ & $1.21 \pm 0.05$ \\
$\log g_{\mathrm{P}}(\mathrm{cgs})$ & $3.2 \pm 0.1$ & $2.95 \pm 0.05$ & $2.83 \pm 0.09$ & $3.42 \pm 0.05$ & $3.26 \pm 0.04$ \\
$\rho_{\mathrm{P}}\left(\rho_{\mathrm{J}}\right)$ & $0.5 \pm 0.2$ & $0.32 \pm 0.04$ & $0.31 \pm 0.08$ & $1.2 \pm 0.2$ & $0.66 \pm 0.07$ \\
$a(\mathrm{AU})$ & $0.0363 \pm 0.0007$ & $0.0449 \pm 0.0007$ & $0.0449 \pm 0.0008$ & $0.0628 \pm 0.0007$ & $0.0345 \pm 0.0007$ \\
$e$ & $<0.058$ & $<0.017$ & $<0.18$ & $<0.096$ & $<0.17$ \\
$T_{\mathrm{eq}}(\mathrm{K})$ & $1600 \pm 80$ & $1400 \pm 30$ & $1480 \pm 60$ & $1100 \pm 25$ & $1790 \pm 40$ \\
$\sigma_{\mathrm{jit}}\left(\mathrm{km} \mathrm{s}{ }^{-1}\right)$ & $0.0225,0.0205$ & $0.0115,0$ & $0.062,0.014$ & 0.0165 & 0 \\
\hline
\end{tabular}

Notes. The UTC time of mid-transit, $T_{\mathrm{c}}$, is given as HJD-2 450000 . Upper limits on the eccentricity, $e$, are $95 \%$ confidence limits. Values in parantheses are standard errors in the final digit. $T_{\mathrm{eq}}$ is the planet equilibrium temperature assuming zero albedo. Where two values of the jitter $\left(\sigma_{\mathrm{jit}}\right)$ are given, these apply to radial velocity measurements taken before and after the upgrade to the CORALIE spectrograph, respectively.

Table 7. Kinematics and distances.

\begin{tabular}{lrrlrrr}
\hline \hline Star & $\mu_{\alpha}[\mathrm{mas} / \mathrm{yr}]$ & $\mu_{\delta}[\mathrm{mas} / \mathrm{yr}]$ & $d[\mathrm{pc}]$ & $U\left[\mathrm{~km} \mathrm{~s}^{-1}\right]$ & $V\left[\mathrm{~km} \mathrm{~s}^{-1}\right]$ & $W\left[\mathrm{~km} \mathrm{~s}^{-1}\right]$ \\
\hline WASP-119 & $22.1 \pm 2.1$ & $16.0 \pm 1.8$ & $333 \pm 29$ & $28 \pm 4$ & $-10 \pm 3$ & $12 \pm 3$ \\
WASP-124 & $-2.0 \pm 1.3$ & $-14.4 \pm 1.0$ & $433 \pm 11$ & $-16 \pm 2$ & $-16 \pm 2$ & $12 \pm 2$ \\
WASP-126 & $62.0 \pm 1.1$ & $52.9 \pm 0.9$ & $234 \pm 15$ & $64 \pm 5$ & $-47 \pm 3$ & $14 \pm 2$ \\
WASP-129 & $11.8 \pm 1.3$ & $0.7 \pm 1.8$ & $246 \pm 7$ & $-27 \pm 2$ & $-0 \pm 1$ & $18 \pm 2$ \\
WASP-133 & $9.3 \pm 1.5$ & $-7.8 \pm 1.0$ & $547 \pm 21$ & $24 \pm 3$ & $-8 \pm 3$ & $2 \pm 3$ \\
\hline
\end{tabular}

Notes. Proper motions are from Smart (2013).

Science Fundation (SNF). M. Gillon and E. Jehin are FNRS Research Associates. The authors thank the anonymous referee for helpful comments which have improved the quality of this paper.

\section{References}

Anderson, D. R., Collier Cameron, A., Gillon, M., et al. 2012, MNRAS, 422, 1988

Asplund, M., Grevesse, N., Sauval, A. J., \& Scott, P. 2009, ARA\&A, 47, 481

Bakos, G., Noyes, R. W., Kovács, G., et al. 2004, PASP, 116, 266

Bakos, G. Á., Csubry, Z., Penev, K., et al. 2013, PASP, 125, 154

Becker, J. C., Vanderburg, A., Adams, F. C., Rappaport, S. A., \& Schwengeler, H. M. 2015, ApJ, 812, L18

Bessell, M. S. 2005, ARA\&A, 43, 293

Brewer, J. M., Fischer, D. A., Basu, S., Valenti, J. A., \& Piskunov, N. 2015, ApJ 805,126

Claret, A. 2000, A\&A, 363, 1081

Claret, A. 2004, A\&A, 428, 1001

Coşkunoğlu, B., Ak, S., Bilir, S., et al. 2011, MNRAS, 412, 1237

Collier Cameron, A., Bouchy, F., Hébrard, G., et al. 2007, MNRAS, 375, 951

Deal, M., Richard, O., \& Vauclair, S. 2015, A\&A, 584, A105

Doyle, A. P., Smalley, B., Maxted, P. F. L., et al. 2013, MNRAS, 428, 3164

Doyle, A. P., Davies, G. R., Smalley, B., Chaplin, W. J., \& Elsworth, Y. 2014, MNRAS, 444, 3592

Gillon, M., Smalley, B., Hebb, L., et al. 2009, A\&A, 496, 259

Gillon, M., Jehin, E., Magain, P., et al. 2011, in EPJ Web Conf., 11, 06002

Gonzalez, G. 2015, MNRAS, 446, 1020

Holmberg, J., Nordström, B., \& Andersen, J. 2007, A\&A, 475, 519
Howell, S. B., Sobeck, C., Haas, M., et al. 2014, PASP, 126, 398 Huélamo, N., Figueira, P., Bonfils, X., et al. 2008, A\&A, 489, L9 Jehin, E., Gillon, M., Queloz, D., et al. 2011, The Messenger, 145, 2 Jordi, C., Gebran, M., Carrasco, J. M., et al. 2010, A\&A, 523, A48 Kervella, P., Thévenin, F., Di Folco, E., \& Ségransan, D. 2004, A\&A, 426, 297 Lendl, M., Anderson, D. R., Collier-Cameron, A., et al. 2012, A\&A, 544, A72 Maxted, P. F. L., Anderson, D. R., Collier Cameron, A., et al. 2010, PASP, 122, 1465

Maxted, P. F. L., Anderson, D. R., Collier Cameron, A., et al. 2011, PASP, 123, 547

Maxted, P. F. L., Serenelli, A. M., \& Southworth, J. 2015a, A\&A, 575, A36 Maxted, P. F. L., Serenelli, A. M., \& Southworth, J. 2015b, A\&A, 577, A90 Monet, D. G., Levine, S. E., Canzian, B., et al. 2003, AJ, 125, 984

Neveu-VanMalle, M., Queloz, D., Anderson, D. R., et al. 2016, A\&A, 586, A93 Pepper, J., Stanek, K. Z., Pogge, R. W., et al. 2008, AJ, 135, 907

Pollacco, D. L., Skillen, I., Collier Cameron, A., et al. 2006, PASP, 118, 1407 Pollacco, D., Skillen, I., Collier Cameron, A., et al. 2008, MNRAS, 385, 1576 Queloz, D., Henry, G. W., Sivan, J. P., et al. 2001, A\&A, 379, 279

Rauer, H., Catala, C., Aerts, C., et al. 2014, Exp. Astron., 38, 249

Ricker, G. R., et al. 2015, J. Astronomical Telescopes, Instruments, and Systems, 1,014003

Seager, S., \& Mallén-Ornelas, G. 2003, ApJ, 585, 1038

Sestito, P., \& Randich, S. 2005, A\&A, 442, 615

Skrutskie, M. F., Cutri, R.M., Stiening, R., et al. 2006, AJ, 131, 1163

Smart, R. L. 2013, VizieR Online Data Catalog: I/324

Southworth, J., Wheatley, P. J., \& Sams, G. 2007, MNRAS, 379, L11

Steigman, G. 2010, J. Cosmol. Astropart. Phys., 4, 29

Weiss, A., \& Schlattl, H. 2008, Ap\&SS, 316, 99 